\title{
Bayesian Estimations on the Exponentiated Distribution Family with Type-II Right Censoring
}

\author{
Yongku Kim ${ }^{1, a}$, Suk-Bok Kang ${ }^{a}$, Jung-In Seo ${ }^{a}$ \\ ${ }^{a}$ Department of Statistics, Yeungnam University
}

\begin{abstract}
Exponentiated distribution has been used in reliability and survival analysis especially when the data is censored. In this paper, we derive Bayesian estimation of the shape parameter, reliability function and failure rate function in the exponentiated distribution family based on Type-II right censored data. We here consider conjugate prior and noninformative prior and corresponding posterior distributions are obtained. As an illustration, the mean square errors of the estimates are computed. Comparisons are made between these estimators using Monte Carlo simulation study.

Keywords: Exponentiated distribution, Type-II right censoring, reliability, Bayesian estimation.
\end{abstract}

\section{Introduction}

For a random variable with probability density function $u(x)$ and cumulative distribution function $U(x)$, its exponentiated distribution has the probability density function(pdf), and cumulative distribution function(cdf), given respectively, by

$$
f(x ; \lambda)=\lambda U(x)^{\lambda-1} u(x)
$$

and

$$
F(x ; \lambda)=[U(x)]^{\lambda}
$$

where $\lambda>0$ is shape parameter of interest.

For the special case $\lambda=1$, this is the original one. For reliability and survival analysis, a half logistic distribution or a half triangle distribution can be considered. These have been used extensively in reliability and survival analysis particularly when the data is censored. For the half logistic distribution and the half triangle distribution,

$$
U(x)=\left[\frac{1-e^{-\frac{x}{\sigma}}}{1+e^{-\frac{x}{\sigma}}}\right], \quad x>0, \sigma>0
$$

and

$$
U(x)=\left[1-\left(1-\frac{x}{\sigma}\right)^{2}\right], \quad 0<x<\sigma, \sigma>0,
$$

\footnotetext{
${ }^{1}$ Corresponding author: Professor, Department of Statistics, Yeungnam University, Gyeongsan 712-749, Korea.

E-mail: ykkim@yu.ac.kr
} 
respectively, where $\sigma$ is a scale parameter. Inferences for the half logistic distribution were discussed by several authors. Balakrishnan and Puthenpura (1986) introduced the best linear unbiased estimators of location and scale parameters of the half logistic distribution through linear functions of order statistics. Balakrishnan and Wong (1991) obtained approximate maximum likelihood estimates(AMLEs) for the location and scale parameters of the half logistic distribution with Type-II right censored sample. Recently, Kang et al. (2008) derived the AMLEs and maximum likelihood estimator of the scale parameter in a half logistic distribution based on progressively Type-II censored samples. A triangle distribution was applied to a kernel function in non-parametric density estimation. Johnson (1997) studied the possibility of using the more intuitively obvious triangular distribution as a proxy for the beta distribution. Some properties of the triangular distribution was studied by Balakrishnan and Nevzorov (2003).

From (1.1) and (1.2), the reliability and failure rate function of exponentiated distribution family (EDF) are obtained, respectively, by

$$
R(t)=1-[U(t)]^{\lambda}
$$

and

$$
h(t)=\frac{f(t)}{R(t)}=\frac{\lambda U(t)^{\lambda-1} u(t)}{1-[U(t)]^{\lambda}}
$$

respectively.

In Bayesian estimation, we consider two types of loss functions. The first is the squared error loss function (quadratic loss) which is symmetrical, and associates equal importance to the losses due to overestimation and underestimation of equal magnitude. But in life testing and reliability problems, the nature of losses are not always symmetric. For example, if an overestimate is usually much more serious than an underestimate, the use of a symmetrical loss function might be inappropriate. As a useful alternative to the squared error loss function, the second is the linex (linear-exponential) loss function which is asymmetric. It was introduced by Varian (1975) became popular due to Zellner (1986). The linex loss function may be expressed a $l(\Delta) \propto \exp (c \Delta)-c \Delta-1, c \neq 0$, where $\Delta=\hat{\theta}-\theta$. The sign and magnitude of the shape parameter $c$ reflects the direction and degree of asymmetry, respectively. When $c$ is positive, the overestimation is more serious than underestimation and the situation is reverse when $c$ is negative. If $c$ tends to zero, the linex loss function tends to squared error loss function. By Zellner (1986), the Bayes estimator of $\theta$, denoted by $\hat{\theta}_{l}$ under the linex loss function is given by $\hat{\theta}_{l}=-1(1 / c) \log \left\{E_{\pi}[\exp (-c \theta)]\right\}$, provided that the expectation $E_{\pi}[\exp (-c \theta)]$ exists and is finite.

\section{Maximum Likelihood Estimation}

First, we derive the maximum likelihood estimator of the shape parameter and reliability function under the Type-II right censoring, where the test terminates as soon as the $r^{\text {th }}$ item fails $(r<n)$. Let $x_{1}, \ldots, x_{r}$ denote the observed failure times for first $r$ components from an exponentiated distribution having a pdf $f(x ; \lambda)$ and $x_{(1)}, \ldots, x_{(r)}$ denote corresponding order statistics of the random sample $x_{1}, \ldots, x_{r}$. Then, the likelihood function of $r$ failure items is given by

$$
L(\lambda)=\frac{r !}{(n-r) !} \lambda^{r} \prod_{i=1}^{r}\left(u_{i}\right) U_{i}^{\lambda-1}\left(1-U_{r}^{\lambda}\right)^{n-r},
$$


where $u_{i}=u\left(x_{(i)}\right), U_{i}=U\left(x_{(i)}\right)$ and $U_{r}=U\left(x_{(r)}\right)$. If $r=n$, then Equation (2.1) reduces to complete data. In general, the original distribution has its own parameter, say $\sigma$. That is, $U(x)=U(x ; \sigma)$. For now, we assume that the parameter $\sigma$ is known and left to be dealt with later.

Form (2.1), the natural logarithm of the likelihood function is given by

$$
\log L(\lambda)=K+r \log \lambda+(\lambda-1) \sum_{i=1}^{r} \log U_{i}+(n-r) \log \left(1-U_{r}^{\lambda}\right)
$$

where $K$ is a constant. The MLE of $\lambda$, denote by $\hat{\lambda}$, is given by

$$
\hat{\lambda}=\frac{r}{(n-r)\left(U_{r}^{-\hat{\lambda}}-1\right)^{-1} \log U_{r}-\sum_{i=1}^{r} \log U_{i}} .
$$

This equation is in implicit form, so it may be subsequently solved with a numerical method such as Newton-Raphson or Bisection. The MLE of reliability function $R(t)$ may be obtained by replacing $\lambda$ by $\hat{\lambda}$ in Equation (1.3), then the MLE of the cumulative failure rate function $H(t)=-\log R(t)$ can be obtained.

\section{Bayesian Estimation}

The natural family of conjugate prior for $\lambda$ is a gamma distribution with pdf

$$
\pi(\lambda)=\frac{\beta^{\alpha}}{\Gamma(\alpha)} \lambda^{\alpha-1} e^{-\beta \lambda}, \quad \alpha>0, \beta>0 .
$$

Applying Bayes theorem, we obtain from Equation (2.1) and (3.1) the posterior density of $\lambda$ as

$$
\pi(\lambda \mid \mathbf{x})=\frac{T^{r+\alpha}}{M_{1} \Gamma(r+\alpha)} \lambda^{r+\alpha-1} e^{-T \lambda}\left(1-U_{r}^{\lambda}\right)^{n-r},
$$

where $M_{1}=\sum_{j=0}^{n-r}(-1)^{j}\left(\begin{array}{c}n-r \\ j\end{array}\right)\left(1+j V_{r} / T\right)^{-(r+\alpha)}, T=\beta+\sum_{i=1}^{r} V_{i}, V_{r}=\log U_{r}^{-1}$ and $V_{i}=\log U_{i}^{-1}$. Refer to Appendix for detailed proofs in this section.

\subsection{Estimation of shape parameter $\lambda$}

The Bayes estimator of $\lambda$ under squared error loss function is given by

$$
\hat{\lambda}_{s}=\frac{r+\alpha}{T} \frac{M_{2}}{M_{1}}
$$

where $M_{2}=\sum_{j=0}^{n-r}(-1)^{j}\left(\begin{array}{c}n-r \\ j\end{array}\right)\left(1+j V_{r} / T\right)^{-(r+\alpha+1)}$.

The Bayes estimator of $\lambda$ under linex loss function is given by

$$
\hat{\lambda}_{l}=\frac{1}{c} \log \left(\frac{M_{1}}{M_{3}}\right)
$$

where $M_{3}=\sum_{j=0}^{n-r}(-1)^{j}\left(\begin{array}{c}n-r \\ j\end{array}\right)\left(1+\left(c+j V_{r}\right) / T\right)^{-(r+\alpha)}$ and $c$ is the scale parameter of linex loss function. 
The $100(1-\alpha) \%$ HPD credible interval $\left(l_{\lambda}, u_{\lambda}\right)$ of $\lambda$ is given by the simultaneous solution of the equations

$$
\begin{aligned}
1-\alpha= & \sum_{j=0}^{n-r}(-1)^{j}\left(\begin{array}{c}
n-r \\
j
\end{array}\right) \frac{1}{M_{1}}\left(\frac{T}{T-j \log U_{r}}\right)^{r+\alpha} \\
& \times\left[\Gamma_{I}\left(\left(T-j \log U_{r}\right) u_{\lambda}, r+\alpha\right)-\Gamma_{I}\left(\left(T-j \log U_{r}\right) l_{\lambda}, r+\alpha\right)\right]
\end{aligned}
$$

and

$$
\left(\frac{u_{\lambda}}{l_{\lambda}}\right)^{r+\alpha}\left(\frac{1-U_{r}^{u_{\lambda}}}{1-U_{r}^{l_{\lambda}}}\right)^{n-r}=e^{T\left(u_{\lambda}-l_{\lambda}\right)}
$$

where $\Gamma_{I}(a, b)=1 / \Gamma(b) \int_{0}^{a} z^{b-1} e^{-z} d z$, the incomplete gamma function.

\subsection{Estimation of reliability function $R$}

Consider the reliability function $R=R(t)$ is parameter itself. Replacing $\lambda$ in terms of $R$ by that of (3.1), we obtain posterior density function of $R$ as

$$
\pi(R \mid \mathbf{x})=\frac{P^{r+\alpha}}{M_{1} \Gamma(r+\alpha)}\left[G_{1}(R)\right]^{r+\alpha-1} \exp \left[(1-P) G_{1}(R)\right]\left(1-U_{r}^{\frac{G_{1}(R)}{V_{t}}}\right)^{n-r},
$$

where $G_{1}(R)=\log (1-R)^{-1}$ and $P=T / V_{t}$.

Assuming the quadratic loss is appropriate, the Bayes estimator of reliability function $R$ is

$$
\hat{R}_{s}=\frac{M_{4}}{M_{1}}
$$

where $M_{4}=\sum_{j=0}^{n-r}(-1)^{j}\left(\begin{array}{c}n-r \\ j\end{array}\right)\left[\left(1+j V_{r} / T\right)^{-(r+\alpha)}-\left\{1+\left(j V_{r}+V_{t}\right) / T\right\}^{-(r+\alpha)}\right]$.

Under linex loss function, the Bayes estimator of $R$ is

$$
\hat{R}_{l}=1-\frac{1}{c} \log \left(\frac{M_{5}}{M_{1}}\right)
$$

where $M_{5}=\sum_{i=0}^{\infty} c^{i} / i ! \sum_{j=0}^{n-r}(-1)^{j}\left(\begin{array}{c}n-r \\ j\end{array}\right)\left\{1+\left(j V_{r}+i V_{t}\right) / T\right\}^{-(r+\alpha)}$.

\subsection{Estimation of cumulative failure rate function $H$}

To derive the Bayes estimator of the cumulative failure rate function $H(t)=-\log R(t)$, we first obtain the posterior density function of $H=H(t)$, which can be given by

$$
\pi(H \mid \mathbf{x})=\frac{P^{r+\alpha}}{M_{1} \Gamma(r+\alpha)} \frac{e^{-H}}{1+e^{-H}}\left[G_{2}(H)\right]^{r+\alpha-1} \exp \left[P G_{2}(H)\right]\left(1-U_{r}^{G_{2}(H) / V_{t}}\right)^{n-r},
$$

where $G_{2}(H)=\log \left(1-e^{-H}\right)^{-1}$.

The Bayes estimator of $H$ relative to quadratic loss is

$$
\hat{H}_{s}=\frac{M_{6}}{M_{1}},
$$


where $M_{6}=\sum_{i=1}^{\infty} \sum_{j=0}^{n-r}(-1)^{j} / i\left(\begin{array}{c}n-r \\ j\end{array}\right)\left\{1+\left(j V_{r}+i V_{t}\right) / T\right\}^{-(r+\alpha)}$.

When the linex loss function is appropriate, the Bayes estimator of $H$ is

$$
\hat{H}_{l}=\frac{1}{c} \log \left(\frac{M_{1}}{M_{7}}\right),
$$

where $M_{7}=\sum_{i=0}^{c} \sum_{j=0}^{n-r}(-1)^{i+j}\left(\begin{array}{c}n-r \\ j\end{array}\right)\left(\begin{array}{c}c \\ i\end{array}\right)\left\{1+\left(j V_{r}+i V_{t}\right) / T\right\}^{-(r+\alpha)}$.

\subsection{Noninformative prior for $\lambda$}

For the situation where no prior information about the shape parameter $\lambda$ is available, one may use the quasi density as given by

$$
\pi(\lambda)=\frac{1}{\lambda^{d}}, \quad \lambda>0, d>0
$$

This contains Jeffery's noninformative prior as a special case when $d=1$. It follows, from (2.1) and (3.4), that the posterior distribution of $\lambda$ is given by

$$
\pi(\lambda \mid \mathbf{x})=\frac{Q^{r-d+1}}{N_{1} \Gamma(r-d+1)} \lambda^{r-d} e^{-T \lambda}\left(1-U_{r}^{\lambda}\right)^{n-r},
$$

where $N_{1}=\sum_{j=0}^{n-r}(-1)^{j}\left(\begin{array}{c}n-r \\ j\end{array}\right)\left(1+j V_{r} / Q\right)^{-(r-d+1)}$ and $Q=\sum_{i=1}^{r} V_{i}$.

The Bayes estimator of $\lambda$ under squared error loss function is given by

$$
\tilde{\lambda}_{s}=\frac{r-d+1}{Q} \frac{N_{2}}{N_{1}},
$$

where $N_{2}=\sum_{j=0}^{n-r}(-1)^{j}\left(\begin{array}{c}n-r \\ j\end{array}\right)\left(1+j V_{r} / Q\right)^{-(r-d+2)}$.

The Bayes estimator of $\lambda$ under linex loss function is given by

$$
\tilde{\lambda}_{l}=\frac{1}{c} \log \left(\frac{N_{1}}{N_{3}}\right),
$$

where $N_{3}=\sum_{j=0}^{n-r}(-1)^{j}\left(\begin{array}{c}n-r \\ j\end{array}\right)\left\{1+\left(c+j V_{r}\right) / Q\right\}^{-(r-d+1)}$ and $c$ is the scale parameter of linex loss function.

The $100(1-\alpha) \%$ HPD credible interval $\left(l_{\lambda}, u_{\lambda}\right)$ of $\lambda$ is given by the simultaneous solution of the equations

$$
\begin{aligned}
1-\alpha= & \sum_{j=0}^{n-r}(-1)^{j}\left(\begin{array}{c}
n-r \\
j
\end{array}\right) \frac{1}{N_{1}}\left(\frac{Q}{Q-j \log U_{r}}\right)^{r-d+1} \\
& \times\left[\Gamma_{I}\left(\left(Q-j \log U_{r}\right) u_{\lambda}, r-d+1\right)-\Gamma_{I}\left(\left(Q-j \log U_{r}\right) l_{\lambda}, r-d+1\right)\right]
\end{aligned}
$$

and

$$
\left(\frac{u_{\lambda}}{l_{\lambda}}\right)^{r-d+1}\left(\frac{1-U_{r}^{u_{\lambda}}}{1-U_{r}^{l_{\lambda}}}\right)^{n-r}=e^{Q\left(u_{\lambda}-l_{\lambda}\right)},
$$

where $\Gamma_{I}(a, b)=1 / \Gamma(b) \int_{0}^{a} z^{b-1} e^{-z} d z$, the incomplete gamma function. Similarly, we can estimate the reliability function $R$ and the cumulative failure rate function $H$. 


\subsection{Estimation of the distribution $U(x)$}

If the parameter $\sigma$ of original cdf $U(x)$ is known at the beginning we can consider a joint prior distribution of $(\lambda, \sigma)$ and then perform a fully Bayesian inference using a Markov chain Monte Carlo(MCMC) algorithm. We simply estimate the parameter $\sigma$ and then plug it in since the shape parameter $\lambda$ is the parameter of interest. The nuisance parameter $\sigma$ can be estimated by maximizing its marginal likelihood,

$$
L(\sigma)=\int f(\mathbf{x} \mid \sigma, \lambda) \pi(\lambda) d \lambda
$$

That is,

$$
\hat{\sigma}=\underset{\sigma>0}{\arg \max } L(\sigma)
$$

Note that the marginal likelihood of $\sigma$ can be obtained by

$$
\frac{\Gamma(r+\alpha)}{T^{r+\alpha}} \sum_{j=0}^{n-r}(-1)^{j}\left(\begin{array}{c}
n-r \\
j
\end{array}\right)\left(1+\frac{j V_{r}}{T}\right)^{-(r+\alpha)}
$$

or

$$
\frac{\Gamma(r-d+1)}{Q^{r-d+1}} \sum_{j=0}^{n-r}(-1)^{j}\left(\begin{array}{c}
n-r \\
j
\end{array}\right)\left(1+\frac{j V_{r}}{Q}\right)^{-(r-d+1)} .
$$

The variance estimation of $\lambda$ may require adjustment to allow proper account for the uncertainty caused by estimating $\sigma$ because of

$$
\operatorname{Var}(\lambda \mid \mathbf{x})=E_{\sigma \mid \mathbf{x}}[\operatorname{Var}(\lambda \mid \mathbf{x}, \sigma)]+\operatorname{Var}_{\sigma \mid \mathbf{x}}[E(\lambda \mid \mathbf{x}, \sigma)] .
$$

\section{Illustrative Examples}

In this section, we present two examples to illustrate previously discussed estimation methods.

\subsection{Real data}

Mann and Fertig (1973) give failure times to airplane components subjected to a life test. The exponentiated half logistic distribution has often been found as a suitable model in such situations. The data are Type-II censored: 13 components were placed on test and the test was terminated at time of failure. Failure times (in hours) of the 10 components that failed were given as

$$
\begin{array}{llllllllll}
0.22 & 0.50 & 0.88 & 1.00 & 1.32 & 1.33 & 1.54 & 1.76 & 2.50 & 3.00
\end{array}
$$

In this example, we assume that underlying distribution of this data is an exponentiated half logistic distribution based on Type-II censoring (i.e., $n=13$ and $r=10$ ). From (2.2), the MLEs $\hat{\lambda}=1.54991$, $\hat{R}(t=0.3)=0.947761$ and $\hat{H}(t=0.3)=0.05365$ are obtained. To employ the Newton-Raphson method, the second derivatives of the log-likelihood function are required; however, these may be complicated and the Bisection method is used since it is based on halving the interval. Using the formulae presented in Section 3, the Bayes estimators of $\lambda, R(t=0.3)$ and $H(t=0.3)$ are calculated under two types of loss functions such as the squared error loss function and the linex loss function. These values are given in Table 1 and Table 2. The 95\% HPD credible intervals for the shape parameter $\lambda$ based on gamma prior and quasi prior are $(1.2139,1.9547)$ and $(1.1231,1.9809)$, respectively. 
Table 1: Bayes estimators using the gamma prior with $\alpha=3.0$ and $\beta=2.5$.

\begin{tabular}{cccccc}
\hline \hline$\tilde{\lambda}_{S}$ & $\tilde{\lambda}_{L(c=0.5)}$ & $\tilde{R}_{S}(t=0.3)$ & $\tilde{R}_{L(c=5)}(t=0.3)$ & $\tilde{H}_{S}(t=0.3)$ & $\tilde{H}_{L(c=5)}(t=0.3)$ \\
\hline 1.59129 & 1.55297 & 0.93767 & 0.93331 & 0.06554 & 0.0602 \\
\hline \hline
\end{tabular}

Table 2: Bayes estimators using the quasi prior with $d=2$.

\begin{tabular}{cccccc}
\hline \hline$\tilde{\lambda}_{S}$ & $\tilde{\lambda}_{L(c=0.5)}$ & $\tilde{R}_{S}(t=0.3)$ & $\tilde{R}_{L(c=5)}(t=0.3)$ & $\tilde{H}_{S}(t=0.3)$ & $\tilde{H}_{L(c=5)}(t=0.3)$ \\
\hline 1.58842 & 1.53804 & 0.93265 & 0.92544 & 0.07154 & 0.06369 \\
\hline \hline
\end{tabular}

Table 3: Relative mean squared errors for the estimators of the shape parameter $\lambda, R(t)$ and $H(t)$ when prior is gamma density with $\alpha=6.0$ and $\beta=1.5$.

\begin{tabular}{cc|ccc|ccc|ccc}
\hline \hline$n$ & $r$ & $\hat{\lambda}$ & $\tilde{\lambda}_{S}$ & $\tilde{\lambda}_{L(c=3)}$ & $\hat{R}$ & $\tilde{R}_{S}$ & $\tilde{R}_{L(c=5)}$ & $\hat{H}$ & $\tilde{H}_{S}$ & $\tilde{H}_{L(c=2)}$ \\
\hline \multirow{2}{*}{20} & 20 & 0.1437 & 0.1567 & 0.0886 & 0.0010 & 0.0005 & 0.0003 & 0.0016 & 0.0007 & 0.0010 \\
& 15 & 0.1324 & 0.1477 & 0.0938 & 0.0010 & 0.0005 & 0.0003 & 0.0014 & 0.0006 & 0.0009 \\
\hline \multirow{3}{*}{30} & 30 & 0.0734 & 0.0900 & 0.0442 & 0.0006 & 0.0003 & 0.0002 & 0.0008 & 0.0004 & 0.0006 \\
& 25 & 0.0701 & 0.0869 & 0.0460 & 0.0005 & 0.0003 & 0.0002 & 0.0008 & 0.0004 & 0.0006 \\
& 20 & 0.0664 & 0.0838 & 0.0484 & 0.0005 & 0.0003 & 0.0002 & 0.0008 & 0.0004 & 0.0006 \\
\hline \multirow{3}{*}{40} & 40 & 0.0455 & 0.0590 & 0.0257 & 0.0004 & 0.0002 & 0.0002 & 0.0005 & 0.0003 & 0.0004 \\
& 35 & 0.0441 & 0.0576 & 0.0265 & 0.0003 & 0.0002 & 0.0002 & 0.0005 & 0.0003 & 0.0004 \\
& 30 & 0.0426 & 0.0562 & 0.0275 & 0.0003 & 0.0002 & 0.0002 & 0.0005 & 0.0003 & 0.0004 \\
\hline \hline
\end{tabular}

Table 4: Relative mean squared errors for the estimators of the shape parameter $\lambda, R(t)$ and $H(t)$ when prior is quasi density with $d=0.4$.

\begin{tabular}{cc|ccc|ccc|ccc}
\hline \hline$n$ & $r$ & $\hat{\lambda}$ & $\tilde{\lambda}_{S}$ & $\tilde{\lambda}_{L(c=3)}$ & $\hat{R}$ & $\tilde{R}_{S}$ & $\tilde{R}_{L(c=5)}$ & $\hat{H}$ & $\tilde{H}_{S}$ & $\tilde{H}_{L(c=2)}$ \\
\hline \multirow{2}{*}{20} & 20 & 0.1437 & 0.2468 & 0.1352 & 0.0010 & 0.0008 & 0.0004 & 0.0016 & 0.0010 & 0.0015 \\
& 15 & 0.1324 & 0.2322 & 0.1432 & 0.0010 & 0.0007 & 0.0003 & 0.0014 & 0.0009 & 0.0014 \\
\hline \multirow{3}{*}{30} & 30 & 0.0734 & 0.1206 & 0.0618 & 0.0006 & 0.0004 & 0.0003 & 0.0008 & 0.0006 & 0.0008 \\
& 25 & 0.0701 & 0.1163 & 0.0643 & 0.0005 & 0.0004 & 0.0003 & 0.0008 & 0.0005 & 0.0008 \\
& 20 & 0.0664 & 0.1122 & 0.0676 & 0.0005 & 0.0004 & 0.0002 & 0.0008 & 0.0005 & 0.0007 \\
\hline \multirow{3}{*}{40} & 40 & 0.0455 & 0.0728 & 0.0342 & 0.0004 & 0.0003 & 0.0002 & 0.0005 & 0.0004 & 0.0005 \\
& 35 & 0.0441 & 0.0710 & 0.0352 & 0.0003 & 0.0003 & 0.0002 & 0.0005 & 0.0004 & 0.0005 \\
& 30 & 0.0426 & 0.0692 & 0.0364 & 0.0003 & 0.0003 & 0.0002 & 0.0005 & 0.0003 & 0.0005 \\
\hline \hline
\end{tabular}

\subsection{Simulation assessment}

To compare the performance of the Bayes estimators of $\lambda, R(t)$ and $H(t)$ under the squared error loss function and the linex loss function, we simulated the mean squared errors of all proposed estimators through Monte Carlo simulation method. The prior parameters chosen $(\alpha, \beta)=(6.0,1.5)$, that yield the generated value of $\lambda=3.54714$ as the true value. The true values of $R(t)$ and $H(t)$ at time $t=0.4$ are computed to be $R(t=0.4)=0.79465$ and $H(t=0.4)=0.22985$. Using the true value of $\lambda$, the Type-II censored data from the exponentiated half triangle distribution are generated for sample size $n=20,30,40$ and various Type-II censoring schemes. Using this data, the mean squared errors of the Bayes estimators of $\lambda, R(t)$ and $H(t)$ under the squared error loss function and the linex loss function are simulated by the Monte Carlo method based on 10,000 runs for sample size $n=20,30,40$ and various choices of censoring under Type-II censored samples. These values under the gamma prior and the quasi prior are given in Table 3 and Table 4, respectively. The MLEs of $\lambda$, $R(t)$ and $H(t)$ are compared with Bayes estimators under the squared error loss function and the linex loss function in terms of estimated MSE. The computation of Bayes estimators is more tentative than MLEs. The Bayes estimators under the linex error loss function (that is asymmetric) show an overall better performance than their corresponding MLEs and Bayes estimators under the squared error loss function (that is symmetric); however, Bayes estimators under the squared error loss function provide 
Table 5: Coverage probabilities of the estimators of the shape parameter $\lambda$ when prior is gamma density with $\alpha=6.0$ and $\beta=1.5$.

\begin{tabular}{cc|ccc}
\hline \hline$n$ & $r$ & $\hat{\lambda}$ & $\tilde{\lambda}_{S}$ & $\tilde{\lambda}_{L(c=3)}$ \\
\hline \multirow{2}{*}{20} & 20 & $(0.0341,0.9363)$ & $(0.0387,0.9398)$ & $(0.0421,0.9415)$ \\
& 15 & $(0.0310,0.9215)$ & $(0.0352,0.9372)$ & $(0.0411,0.9389)$ \\
\hline \multirow{2}{*}{30} & 30 & $(0.0398,0.9378)$ & $(0.0424,0.9403)$ & $(0.0451,0.9421)$ \\
& 25 & $(0.0387,0.9324)$ & $(0.0398,0.9372)$ & $(0.0425,0.9418)$ \\
& 20 & $(0.0345,0.9312)$ & $(0.0375,0.9332)$ & $(0.0420,0.9412)$ \\
\hline \multirow{2}{*}{40} & 40 & $(0.0436,0.9413)$ & $(0.0487,0.9476)$ & $(0.0498,0.9487)$ \\
& 35 & $(0.0414,0.9387)$ & $(0.0455,0.9418)$ & $(0.0463,0.9431)$ \\
\hline \hline
\end{tabular}

Table 6: Coverage probabilities of the estimators of the shape parameter $\lambda$ when prior is quasi density with $d=0.4$.

\begin{tabular}{cc|ccc}
\hline \hline$n$ & $r$ & $\hat{\lambda}$ & $\tilde{\lambda}_{S}$ & $\tilde{\lambda}_{L(c=3)}$ \\
\hline \multirow{2}{*}{20} & 20 & $(0.0305,0.9225)$ & $(0.0327,0.9309)$ & $(0.0373,0.9332)$ \\
& 15 & $(0.0287,0.9198)$ & $(0.0310,0.9293)$ & $(0.0352,0.9301)$ \\
\hline \multirow{2}{*}{30} & 30 & $(0.0358,0.9298)$ & $(0.0401,0.9325)$ & $(0.0432,0.9389)$ \\
& 25 & $(0.0338,0.9253)$ & $(0.0379,0.9319)$ & $(0.0412,0.9356)$ \\
& 20 & $(0.0310,0.9219)$ & $(0.0322,0.9312)$ & $(0.0371,0.9329)$ \\
\hline \multirow{2}{*}{40} & 40 & $(0.0397,0.9368)$ & $(0.0437,0.9405)$ & $(0.0446,0.9428)$ \\
& 35 & $(0.0382,0.9315)$ & $(0.0425,0.9388)$ & $(0.0434,0.9401)$ \\
\hline \hline
\end{tabular}

superior results than others for the cumulative failure rate $H(t)$. Table 5 and Table 6 shows that an (informative) gamma prior provides a better match to the target coverage probabilities' 0.05 and 0.95 than a (noninformative) quasi prior especially when the sample size is small.

\section{Concluding Remarks}

In this paper, we present the Bayesian and Non-Bayesian estimator of the shape parameter $\lambda$, reliability function $R(t)$ and cumulative failure rate function $H(t)$ of the exponentiated distribution family with Type-II censoring. Bayes estimators under the squared error loss function and the linex loss function are derived. The MLE's are also obtained through the use of a gamma prior and quasi prior.

\section{Appendix}

1. The posterior density of $\lambda$ :

$$
\pi(\lambda \mid \mathbf{x})=\frac{\lambda^{r} \exp \left(\lambda \sum_{i=1}^{r} \log U_{i}\right)\left(1-U_{r}^{\lambda}\right)^{n-r} \lambda^{\alpha-1} \exp (-\beta \lambda)}{\int_{0}^{\infty} \lambda^{r} \exp \left(\lambda \sum_{i=1}^{r} \log U_{i}\right)\left(1-U_{r}^{\lambda}\right)^{n-r} \lambda^{\alpha-1} \exp (-\beta \lambda) d \lambda},
$$

where

$$
\begin{aligned}
\text { the denominator } & =\int_{0}^{\infty} \lambda^{r+\alpha-1} \exp \left(\lambda\left[\sum_{i=1}^{r} \log U_{i}-\beta\right]\right) \sum_{j=0}^{n-r}(-1)^{j}\left(\begin{array}{c}
n-r \\
j
\end{array}\right) U_{r}^{j \lambda} d \lambda \\
& =\sum_{j=0}^{n-r}(-1)^{j}\left(\begin{array}{c}
n-r \\
j
\end{array}\right) \int_{0}^{\infty} \lambda^{r+\alpha-1} \exp \left(-\lambda\left[\beta-\sum_{i=1}^{r} \log U_{i}-j \log U_{r}\right]\right) d \lambda
\end{aligned}
$$




$$
=\frac{\Gamma(r+\alpha)}{\left(\beta-\sum_{i=1}^{r} \log U_{i}\right)^{r+\alpha}} \sum_{j=0}^{n-r}(-1)^{j}\left(\begin{array}{c}
n-r \\
j
\end{array}\right)\left(1-\frac{j \log U_{r}}{\beta-\sum_{i=1}^{r} \log U_{i}}\right)^{-(r+\alpha)} .
$$

Therefore

$$
\pi(\lambda \mid \mathbf{x})=\frac{\left(\beta-\sum_{i=1}^{r} \log U_{i}\right)^{r+\alpha}}{M_{1} \Gamma(r+\alpha)} \lambda^{r+\alpha-1} e^{-\lambda\left(\beta-\sum_{i=1}^{r} \log U_{i}\right)}\left(1-U_{r}^{\lambda}\right)^{n-r} .
$$

2. Bayes estimator of $\lambda$ under SELF:

$$
\begin{aligned}
\tilde{\lambda}_{S} & =\int_{0}^{\infty} \lambda \frac{\left(\beta-\sum_{i=1}^{r} \log U_{i}\right)^{r+\alpha}}{M_{1} \Gamma(r+\alpha)} \lambda^{r+\alpha-1} e^{-\lambda\left(\beta-\sum_{i=1}^{r} \log U_{i}\right)}\left(1-U_{r}^{\lambda}\right)^{n-r} d \lambda \\
& =\frac{\left(\beta-\sum_{i=1}^{r} \log U_{i}\right)^{r+\alpha}}{M_{1} \Gamma(r+\alpha)} \int_{0}^{\infty} \lambda^{r+\alpha} e^{-\lambda\left(\beta-\sum_{i=1}^{r} \log U_{i}\right)}\left(1-U_{r}^{\lambda}\right)^{n-r} d \lambda \\
& =\frac{\left(\beta-\sum_{i=1}^{r} \log U_{i}\right)^{r+\alpha}}{M_{1} \Gamma(r+\alpha)} \frac{\Gamma(r+\alpha+1)}{\left(\beta-\sum_{i=1}^{r} \log U_{i}\right)^{r+\alpha+1}} \sum_{p=0}^{n-r}\left(\begin{array}{c}
n-r \\
p
\end{array}\right)(-1)^{p}\left(1-\frac{p \log U_{r}}{\beta-\sum_{i=1}^{r} \log U_{i}}\right)^{-(r+\alpha+1)} \\
& =\frac{r+\alpha}{M_{1}\left(\beta-\sum_{i=1}^{r} \log U_{i}\right)} \sum_{p=0}^{n-r}\left(\begin{array}{c}
n-r \\
p
\end{array}\right)(-1)^{p}\left(1-\frac{p \log U_{r}}{\beta-\sum_{i=1}^{r} \log U_{i}}\right)^{-(r+\alpha+1)} .
\end{aligned}
$$

3. Bayes estimator of $\lambda$ under LLF:

$$
\tilde{\lambda}_{L}=-\frac{1}{c} \log E_{\pi}\left[e^{-c \lambda}\right]
$$

where

$$
\begin{aligned}
E_{\pi}\left[e^{-c \lambda}\right] & =\int_{0}^{\infty} e^{-c \lambda} \frac{\left(\beta-\sum_{i=1}^{r} \log U_{i}\right)^{r+\alpha}}{M_{1} \Gamma(r+\alpha)} \lambda^{r+\alpha-1} e^{-\lambda\left(\beta-\sum_{i=1}^{r} \log U_{i}\right)}\left(1-U_{r}^{\lambda}\right)^{n-r} d \lambda \\
& =\frac{\left(\beta-\sum_{i=1}^{r} \log U_{i}\right)^{r+\alpha}}{M_{1} \Gamma(r+\alpha)} \int_{0}^{\infty} \lambda^{r+\alpha-1} e^{-\lambda\left(\beta+c-\sum_{i=1}^{r} \log U_{i}\right)}\left(1-U_{r}^{\lambda}\right)^{n-r} d \lambda \\
& =\frac{\left(\beta-\sum_{i=1}^{r} \log U_{i}\right)^{r+\alpha}}{M_{1} \Gamma(r+\alpha)} \sum_{p=0}^{n-r}\left(\begin{array}{c}
n-r \\
p
\end{array}\right)(-1)^{p} \frac{\Gamma(r+\alpha)}{\left.\beta+c-\sum_{i=1}^{r} \log U_{i}-p \log U_{r}\right)^{r+\alpha}} \\
& =\frac{\left(\beta-\sum_{i=1}^{r} \log U_{i}\right)^{r+\alpha}}{M_{1} \Gamma(r+\alpha)} \frac{\Gamma(r+\alpha)}{\left(\beta-\sum_{i=1}^{r} \log U_{i}\right)^{r+\alpha}} \sum_{p=0}^{n-r}\left(\begin{array}{c}
n-r \\
p
\end{array}\right)(-1)^{p}\left(1+\frac{c-p \log U_{r}}{\beta-\sum_{i=1}^{r} \log U_{i}}\right)^{-(r+\alpha)} \\
& =\frac{1}{M_{1}} \sum_{p=0}^{n-r}\left(\begin{array}{c}
n-r \\
p
\end{array}\right)(-1)^{p}\left(1+\frac{c-p \log U_{r}}{\beta-\sum_{i=1}^{r} \log U_{i}}\right)^{-(r+\alpha)} .
\end{aligned}
$$

Therefore,

$$
\begin{aligned}
\tilde{\lambda}_{L} & =-\frac{1}{c} \log \frac{1}{M_{1}} \sum_{p=0}^{n-r}\left(\begin{array}{c}
n-r \\
p
\end{array}\right)(-1)^{p}\left(1+\frac{c-p \log U_{r}}{\beta-\sum_{i=1}^{r} \log U_{i}}\right)^{-(r+\alpha)} \\
& =\frac{1}{c} \log \frac{M_{1}}{\sum_{p=0}^{n-r}\left(\begin{array}{c}
n-r \\
p
\end{array}\right)(-1)^{p}\left(1+\frac{c-p \log U_{r}}{\beta-\sum_{i=1}^{i} \log U_{i}}\right)^{-(r+\alpha)}} .
\end{aligned}
$$


4. HPD credible interval of $\lambda$ :

$$
\begin{aligned}
\int_{l}^{u} \pi(\lambda \mid \mathbf{x}) d \lambda= & \int_{l}^{u} \frac{\left(\beta-\sum_{i=1}^{r} \log U_{i}\right)^{r+\alpha}}{M_{1} \Gamma(r+\alpha)} \lambda^{r+\alpha-1} e^{-\lambda\left(\beta-\sum_{i=1}^{r} \log U_{i}\right)}\left(1-U_{r}^{\lambda}\right)^{n-r} d \lambda \\
= & \sum_{j=0}^{n-r}(-1)^{j}\left(\begin{array}{c}
n-r \\
j
\end{array}\right) \frac{1}{M_{1}}\left(\frac{T}{T-j \log U_{r}}\right)^{r+\alpha} \times \frac{1}{\Gamma(r+\alpha)} \int_{\left(T-j \log U_{r}\right) l}^{\left(T-j \log U_{r}\right) u} \lambda^{r+\alpha-1} e^{\lambda} d \lambda \\
= & \sum_{j=0}^{n-r}(-1)^{j}\left(\begin{array}{c}
n-r \\
j
\end{array}\right) \frac{1}{M_{1}}\left(\frac{T}{T-j \log U_{r}}\right)^{r+\alpha} \\
& \times\left[\Gamma_{I}\left(\left(T-j \log U_{r}\right) u, r+\alpha\right)-\Gamma_{I}\left(\left(T-j \log U_{r}\right) l, r+\alpha\right)\right] .
\end{aligned}
$$

5. The posterior density of $R(t)$ :

Since $R=R(t)=1-\left[1-(1-t / \sigma)^{2}\right]^{\lambda}$,

$$
\lambda=\frac{\log (1-R)}{\log \left[1-(1-t / \sigma)^{2}\right]} \quad \text { and } \quad\left|\frac{d R}{d \lambda}\right|^{-1}=-\frac{1}{(1-R) \log U_{t}} .
$$

Therefore,

$$
\begin{aligned}
\pi(R \mid \mathbf{x}) & =\pi(\lambda(R) \mid \mathbf{x})\left|\frac{d R}{d \lambda}\right|^{-1} \\
& =\frac{\left(\beta-\sum_{i=1}^{r} \log U_{i}\right)^{r+\alpha}}{M_{1} \Gamma(r+\alpha)}(\lambda(R))^{r+\alpha-1} e^{-\lambda(R)\left(\beta-\sum_{i=1}^{r} \log U_{i}\right)}\left(1-U_{r}^{\lambda(R)}\right)^{n-r}\left(-\frac{1}{(1-R) \log U_{t}}\right) \\
& =\frac{\left(\beta-\sum_{i=1}^{r} U_{i}\right)^{r+\alpha}}{M_{1} \Gamma(r+\alpha)}\left(\frac{1}{\log U_{t}}\right)^{r+\alpha}\left(\log (1-R)^{-1}\right)^{r+\alpha-1}\left(\frac{1}{1-R}\right)^{\frac{\beta-\sum_{i=1}^{r} \log U_{i}}{\log U_{t}}+1}\left[1-U_{r}^{\frac{\log (1-R)}{\log U_{t}}}\right]^{n-r} \\
& =\frac{T^{r+\alpha}}{M_{1} \Gamma(r+\alpha)}\left(\frac{1}{V_{t}}\right)^{r+\alpha}\left(\log (1-R)^{-1}\right)^{r+\alpha-1}\left(\frac{1}{1-R}\right)^{1-\frac{T}{V_{t}}}\left[1-U_{r}^{\frac{\log (1-R)^{-1}}{V_{t}}}\right]^{n-r}\left[1-U_{r}^{\frac{G_{1}(R)}{V_{t}}}\right]^{n-r}, \\
& =\frac{p^{r+\alpha}}{M_{1} \Gamma(r+\alpha)}\left(G_{1}(R)\right)^{r+\alpha-1} e^{G_{1}(R)(1-p)}\left[1-T^{r}\right.
\end{aligned}
$$

where $V_{t}=\log U_{t}^{-1}, G_{1}(R)=\log (1-R)^{-1}$ and $p=T / V_{t}$.

6. The posterior density of $H(t)$ :

Since $H=H(t)=-\log R(t)$,

$$
R=e^{-H} \quad \text { and } \quad\left|\frac{d H}{d R}\right|^{-1}=R=e^{H}
$$

Therefore,

$$
\begin{aligned}
\pi(H \mid \mathbf{x}) & =\pi(R(H) \mid \mathbf{x})\left|\frac{d H}{d R}\right|^{-1} \\
& =\frac{p^{r+\alpha}}{M_{1} \Gamma(r+\alpha)}\left(\log \left(1-e^{-H}\right)^{-1}\right)^{r+\alpha-1} e^{\left(\log \left(1-e^{-H}\right)^{-1}\right)(1-p)}\left[1-U_{r}^{\frac{\log \left(1-e^{-H}\right)^{-1}}{V_{t}}}\right]^{n-r} e^{H}
\end{aligned}
$$




$$
\begin{aligned}
& =\frac{p^{r+\alpha}}{M_{1} \Gamma(r+\alpha)}\left(G_{2}(H)\right)^{r+\alpha-1}\left(\frac{1}{1+e^{H}}\right)^{1-p}\left[1-U_{r}^{\frac{G_{2}(H)}{V_{t}}}\right]^{n-r} e^{H} \\
& =\frac{p^{r+\alpha}}{M_{1} \Gamma(r+\alpha)}\left(G_{2}(H)\right)^{r+\alpha-1} e^{G_{2}(H) p}\left(\frac{e^{H}}{1+e^{H}}\right)\left[1-U_{r}^{\frac{G_{2}(H)}{V_{t}}}\right]^{n-r} e^{H},
\end{aligned}
$$

where $G_{2}(H)=\log \left(1-e^{-H}\right)^{-1}$.

7. The rest can be proved in a similar way.

\section{References}

Balakrishnan, N. and Nevzorov, V. B. (2003). A Primer on Statistical Distribution, John Willey \& Stone, New York.

Balakrishnan, N. and Puthenpura, S. (1986). Best linear unbiased estimators of location and scale parameters of the half logistic distribution, Journal of statistical Computation and Simulation, 25, 193-204.

Balakrishnan, N. and Wong, K. H. T. (1991). Approximate MLEs for the location and scale parameters of the half logistic distribution with Type-II right-censoring, IEEE Transactions on Reliability, 40, 140-145.

Johnson, D. (1997). The triangular distribution as a proxy for the beta distribution in risk analysis, The Statistician, 46, 387-398.

Kang, S. B., Cho, Y. S. and Han, J. T. (2008). Estimation for the half logistic distribution under progressive Type-II Censoring, Communications of the Korean Statistical Society, 15, 815-823.

Mann, N. R. and Fertig, K. W. (1973). Tables for obtaining confidence bounds and tolerance bounds based on best linear invariant estimates of parameters of the extreme value distribution, Technometrics, 15, 87-101.

Varian, H. R. (1975). A Bayesian approach to real estate assessment. In: S. E. Feinberg and A. Zellner, Eds., Studies in Bayesian Econometrics and Statistics in Honor of Leonard J. Savage, North Holland, Amsterdam, 195-208.

Zellner, A. (1986). Bayesian estimation and prediction using asymmetric loss function, Journal of American Statistical Association, 81, 446-451. 